\title{
Against Geometry: Nonstandard General Relativity
}

\author{
Günter Scharf \\ Physics Institute, University of Zürich, Zürich, Switzerland \\ Email: scharf@physik.uzh.ch
}

Received 27 February 2015; accepted 14 March 2015; published 18 March 2015

Copyright (C) 2015 by author and OALib.

This work is licensed under the Creative Commons Attribution International License (CC BY). http://creativecommons.org/licenses/by/4.0/

(c) (i) Open Access

\section{Abstract}

We show that the Schwarzschild solution can be embedded in a class of nonstandard solutions of the vacuum Einstein's equations with arbitrary rotation curves. These nonstandard solutions have to be taken as physical, if dark matter as needed in the standard theory cannot be found. As a consequence general relativity is considered as a classical field theory in Minkowski space and not as a geometric theory in the sense of Einstein. Assuming an asymptotically flat rotation curve and introducing a material disk into this model we find a matter density in accordance with the TullyFisher relation.

\section{Keywords}

Dark Matter, General Relativity

Subject Areas: Modern Physics

\section{Introduction}

General relativity is a classical gauge theory. This implies that the fundamental fields, as the metric $g_{\mu \nu}$, are not directly observable. Therefore, in investigating gravitational effects it is important to identify the observable quantities which are actually measurable. Every observable is defined by a measuring process, the coordinate system included. A change of the coordinates, although mathematically possible, is dangerous for physical reasons: one may lose the contact with the measurable observables. Therefore, we choose physically defined coordinates once and for all and do not change them. On the scale of galaxies one most important observable is the circular velocity $V(r)$ of stars or gas which can be measured by the Doppler shift of spectral lines ( $r$ is the radius of the circular orbit). This observable plays an important role in the following: we will use it to fix the gauge.

In standard general relativity one is tempted to interpret the metric $g_{\mu v}$ geometrically, for example by using it to measure the circumference of a circle in space. We reject this because, similarly as in electrodynamics, the 
$g_{\mu v}$ are the gravitational potentials and as such they are not observable. A nice way to see this is to consider electrodynamics and general relativity side by side. The electromagnetic fields $F^{\mu v}$ are defined by their effect on the motion of charged test bodies according to the equation of motion

$$
\frac{\mathrm{d}^{2} x^{\mu}}{\mathrm{d} \tau^{2}}=\frac{e}{m} F^{\mu \nu} \frac{\mathrm{d} x_{v}}{\mathrm{~d} \tau}
$$

here the Lorentz force appears on the r.h.s. The corresponding equation of motion for test bodies in a gravitational field is the geodesic equation

$$
\frac{\mathrm{d}^{2} x^{\mu}}{\mathrm{d} \tau^{2}}+\Gamma_{\alpha \beta}^{\mu} \frac{\mathrm{d} x^{\alpha}}{\mathrm{d} \tau} \frac{\mathrm{d} x^{\beta}}{\mathrm{d} \tau}=0
$$

Consequently the Christoffel symbols are the gravitational field strengths. The field equations for the $F^{\mu v}$ are the inhomogeneous Maxwell's equations

$$
\partial_{v} F^{\mu v}=-\mu_{0} j^{\mu}
$$

The field equations for the $\Gamma_{\alpha \beta}^{\mu}$ are Einstein's equations

$$
R_{\mu \nu}=\kappa\left(T_{\mu \nu}-\frac{1}{2} g_{\mu \nu} T_{\alpha}^{\alpha}\right)
$$

These are first order partial differential equations as (1.3), because the Ricci tensor is given by

$$
R_{\mu v}=\partial_{\alpha} \Gamma_{\mu \nu}^{\alpha}-\partial_{v} \Gamma_{\mu \alpha}^{\alpha}+\Gamma_{\alpha \beta}^{\alpha} \Gamma_{\mu \nu}^{\beta}-\Gamma_{\nu \beta}^{\alpha} \Gamma_{\alpha \mu}^{\beta} .
$$

However (1.3) are only four equations for the 6 components of $F^{\mu v}$. The gap is filled by introducing the vector potential

$$
F^{\mu v}=\partial^{\mu} A^{v}-\partial^{v} A^{\mu},
$$

which is a consequence of the homogeneous Maxwell's equations. Similarly (1.4) are only 10 equations for the 40 components of $\Gamma$. The gap is filled by introducing the metric according to

$$
\Gamma_{\beta \gamma}^{\alpha}=\frac{1}{2} g^{\alpha \mu}\left(\partial_{\gamma} g_{\beta \mu}+\partial_{\beta} g_{\mu \gamma}-\partial_{\mu} g_{\beta \gamma}\right)
$$

Since the electromagnetic potentials are not observable quantities, the same must be true for the metric tensor which, therefore, has no direct physical interpretation in general. Consequently, we do not interprete $g_{\mu v}$ geometrically; it is a parametrization of the gravitational field and nothing else. Here we are following Poincaré ([1], p. 50) and consider geometry as a convention. Standard general relativity is based on the fusion of geometry and gravitation, and one has considered this fusion as the most beautiful achievement of the general theory of relativity (W. Pauli, The Theory of Relativity, Dover, p. 148). In the nonstandard theory this fusion is suspended.

In the theoretical analysis one should try to relate the observables to the metric $g_{\mu v}(x)$. The best would be if the metric under certain assumptions can be uniquely expressed by the observables. After the gauge ambiguity has been removed, the gauge is fixed by a physical requirement. Another possibility is to choose the gauge on unphysical grounds, for example by some geometric convention or/and to simplify the solution of the differential equations. This standard approach is dangerous because one might miss some important physics. Our program of fixing the gauge by observables is a sort of inverse procedure compared with standard general relativity where one first calculates a metric by solving Einstein's equations in some special gauge and then determines the observables. Clearly, in standard general relativity one cannot be sure that one finds all physically relevant solutions. Indeed we are going to show that Einstein's equations have vacuum solutions with an asymptotically flat rotation curve $V(r)$. These nonstandard solutions can be used to describe the dark halo of galaxies without introducing hypothetical dark matter. When the recent Xenon-experiment has again not found any signal of dark matter particles [2], one should seriously investigate nonstandard general relativity.

The paper is organized as follows. As preliminaries we first consider the rotation curve in a general spherically symmetric gravitational field. Although this may be known we do not know a good reference. In Section 3 we solve the vacuum Einstein's equations in our general spherically symmetric setting and express the metric 
tensor by the circular velocity $V(r)$. We find a class of nonstandard solutions which contains the Schwarzschild solution as a special case. All these solutions describe different physics if the circular velocities are different. From these vacuum solutions we construct in Section 4 solutions with a disk of ordinary matter by means of the well-known displace, cut, and reflect method. This gives a simple model of a spiral galaxy. Assuming a circular velocity which is constant $=V_{\text {flat }}$ for large $r$, we find a matter density proportional to $V_{\text {flat }}^{4}$. This is in accordance with the Tully-Fisher relation [4] [5]. We close with some concluding remarks about standard and nonstandard general relativity. In particular we discuss the connection with MOND.

\section{The Circular Velocity in General Relativity}

We consider a star moving in an arbitrary static gravitational field. Following Weinberg ([3], Chapter 3/2) we introduce the freely falling coordinate system $\xi^{\alpha}$ of the moving star. In this system the star is at rest and the observer on earth moves with the 4-velocity

$$
u^{\alpha}=\frac{\mathrm{d} \xi^{\alpha}}{\mathrm{d} \tau}=\left(u^{0}, \boldsymbol{u}\right), \quad u^{0}=\left(1-\boldsymbol{V}^{2}\right)^{-1 / 2}, \quad \boldsymbol{u}=u^{0} \boldsymbol{V},
$$

because in the locally inertial coordinates special relativity holds. Here $\tau$ is the proper time

$$
\mathrm{d} \tau^{2}=\eta_{\alpha \beta} \mathrm{d} \xi^{\alpha} \mathrm{d} \xi^{\beta},
$$

$\eta_{\alpha \beta}=\operatorname{diag}(1,-1,-1,-1)$ is the Minkowski tensor and the speed of light is put $=1$. In addition Weinberg introduces a laboratory coordinate system $x^{\mu}$ which in our case is attached to the observers telescope. To simplify the following discussion we assume that the astronomer on earth has corrected his measurements for the motion of the earth with respect to the center of the galaxy, so that we can choose the center of the galaxy as origin of the laboratory coordinate system. Now the star moves on a geodesic ([3], Equation (3.2.3))

$$
\frac{\mathrm{d}^{2} x^{\mu}}{\mathrm{d} \tau^{2}}+\Gamma_{\alpha \beta}^{\mu} \frac{\mathrm{d} x^{\alpha}}{\mathrm{d} \tau} \frac{\mathrm{d} x^{\beta}}{\mathrm{d} \tau}=0
$$

where $\Gamma_{\alpha \beta}^{\mu}$ are the Christoffel symbols of the metric $g_{\mu v}$. The latter is defined by ([3], Equation (3.2.7))

$$
g_{\mu v}=\frac{\partial \xi^{\alpha}}{\partial x^{\mu}} \eta_{\alpha \beta} \frac{\partial \xi^{\beta}}{\partial x^{\nu}} .
$$

Since the local inertial coordinates $\xi^{\alpha}$ can be changed by arbitrary Lorentz transformations, we choose a Lorentz boost such that the two coordinate systems are at rest with respect to each other. Then we have

$$
\frac{\partial \xi^{0}}{\partial x^{j}}=0, \text { and } \quad \frac{\partial \xi^{j}}{\partial x^{0}}=0
$$

for $j=1,2,3$. This leads to

$$
g_{00}=\left(\frac{\partial \xi^{0}}{\partial x^{0}}\right)^{2}, \quad g_{j k}=-\sum_{i=1}^{3} \frac{\partial \xi^{i}}{\partial x^{j}} \frac{\partial \xi^{i}}{\partial x^{k}}
$$

and $g_{0 j}=0$. Now it follows

$$
u^{0}=\frac{\mathrm{d} \xi^{0}}{\mathrm{~d} \tau}=\frac{\partial \xi^{0}}{\partial x^{0}} \frac{\mathrm{d} x^{0}}{\mathrm{~d} \tau}=\sqrt{g_{00}} \frac{\mathrm{d} x^{0}}{\mathrm{~d} \tau} .
$$

In the following we assume that the non-diagonal elements $g_{j k}$ vanish. Then from the invariant

$$
\begin{aligned}
u^{\alpha} \eta_{\alpha \beta} u^{\beta} & =\left(u^{0}\right)^{2}-u^{2}=1=\frac{\partial \xi^{\alpha}}{\partial x^{\mu}} \eta_{\alpha \beta} \frac{\partial \xi^{\beta}}{\partial x^{\nu}} \frac{\mathrm{d} x^{\mu}}{\mathrm{d} \tau} \frac{\mathrm{d} x^{\nu}}{\mathrm{d} \tau} \\
& =g_{\mu \nu} \frac{\mathrm{d} x^{\mu}}{\mathrm{d} \tau} \frac{\mathrm{d} x^{\nu}}{\mathrm{d} \tau}=g_{00}\left(\frac{\mathrm{d} x^{0}}{\mathrm{~d} \tau}\right)^{2}+g_{j j}\left(\frac{\mathrm{d} x^{j}}{\mathrm{~d} \tau}\right)^{2},
\end{aligned}
$$

we find 


$$
\boldsymbol{V}^{2}=-\frac{1}{\left(u^{0}\right)^{2}} g_{j j}\left(\frac{\mathrm{d} x^{j}}{\mathrm{~d} \tau}\right)^{2}=-\sum_{j=1}^{3} \frac{g_{j j}}{g_{00}} \frac{\left(\mathrm{d} x^{j} / \mathrm{d} \tau\right)^{2}}{\left(\mathrm{~d} x^{0} / \mathrm{d} \tau\right)^{2}}
$$

where (2.7) is used.

We want to specialize this for circular motion $r=$ const choosing spherical coordinates $(t, r, \vartheta, \phi)$. Now the geodesic Equation (2.3) for $\mu=1$ reads

$$
\frac{\mathrm{d}^{2} r}{\mathrm{~d} \tau^{2}}+\Gamma_{00}^{1}\left(\frac{\mathrm{d} t}{\mathrm{~d} \tau}\right)^{2}+\Gamma_{11}^{1}\left(\frac{\mathrm{d} r}{\mathrm{~d} \tau}\right)^{2}+\Gamma_{22}^{1}\left(\frac{\mathrm{d} \vartheta}{\mathrm{d} \tau}\right)^{2}+\Gamma_{33}^{1}\left(\frac{\mathrm{d} \phi}{\mathrm{d} \tau}\right)^{2}=0
$$

Taking the circular orbit $r=$ const in the equatorial plane $\vartheta=\pi / 2$, then (2.9) gets simplified to

$$
\left(\frac{\mathrm{d} \phi}{\mathrm{d} \tau}\right)^{2}=-\frac{\Gamma_{00}^{1}}{\Gamma_{33}^{1}}\left(\frac{\mathrm{d} t}{\mathrm{~d} \tau}\right)^{2}
$$

and from (2.8) we finally obtain the important relation

$$
\boldsymbol{V}^{2}=-\frac{g_{33}}{g_{00}}\left(\frac{\mathrm{d} \phi}{\mathrm{d} t}\right)^{2}=\frac{g_{33}}{g_{00}} \frac{\Gamma_{00}^{1}}{\Gamma_{33}^{1}} .
$$

In the following we consider static spherically symmetric metrics of the form

$$
\mathrm{d} s^{2}=g_{\mu \nu} \mathrm{d} x^{\mu} \mathrm{d} x^{v}=e^{a} \mathrm{~d} t^{2}-e^{b} \mathrm{~d} r^{2}-r^{2} e^{c}\left(\mathrm{~d} \vartheta^{2}+\sin ^{2} \vartheta \mathrm{d} \phi^{2}\right)
$$

where $a(r), b(r), c(r)$ are functions of $r$ only. For this metric we have [6]

$$
\begin{gathered}
\Gamma_{00}^{1}=\frac{a^{\prime}}{2} e^{a-b} \\
\Gamma_{33}^{1}=-\left(\frac{r^{2}}{2} c^{\prime}+r\right) e^{c-b}
\end{gathered}
$$

here the prime means $d / d r$. Then (2.11) simply becomes

$$
V^{2}=\frac{a^{\prime}}{c^{\prime}+2 / r} .
$$

It is not hard to integrate the geodesic equations for the metric (2.12) completely. Then one finds that there exist circular orbits for every radius $r>0$ with circular velocity (2.14).

Instead of using $\boldsymbol{V}^{2}(r)$ we can work with the redshift $z(r)$ which is directly measurable. But then the integration of Einstein's equations in the next section becomes more complicated. We are mainly interested to understand the haloes of galaxies where the gravitational fields are very weak. Then the difference between $V$ and $z$ is not relevant, so that the simple Doppler formula is good enough.

\section{Solution of the Vacuum Equation}

The metric functions $a, b, c$ appearing in (2.12) must satisfy differential equations which follow from Einstein's equations. In standard general relativity one puts $c=0$. This is a special choice of gauge which leads to Birkhoff's theorem and the Schwarzschild metric. This works well in the solar system, but obviously not on the galactic scale. The standard way out is to abandon the vacuum equations and assume some hypothetical dark matter. As long as this dark matter is not convincingly recorded one should also study the other possibility of retaining $c(r) \neq 0$. Then the vacuum solution is no longer unique. To fix it uniquely we take the expression (2.14) for the circular velocity $V(r)$ as our nonstandard gauge condition. It is often argued that by a transformation of coordinates $c=0$ can always be achieved. We show at the end of this section (3.20) that one loses the contact to physics in this way.

Since the circular velocity $V(r)$ must be given the theory seems to have less predictive power. What seems to be a weakness is a strength: The asymptotic $V(r)$ cannot be predicted on the basis of the vacuum equations 
alone, the dynamics of the normal matter, that means the detailed structure of the galaxy, must necessarily be taken into account. Indeed a universal asymptotic velocity profile for all galaxies seems not to exist. In addition, only with $c \neq 0$ is it possible to carry out our program to express the metric by the observable $V(r)$. We continue the discussion of the nonstandard gauge in the concluding remarks.

The non-vanishing components of the Ricci tensor for the metric (2.12) are the diagonal elements [6]

$$
\begin{gathered}
R_{t t}=\frac{1}{2} e^{a-b}\left(a^{\prime \prime}+\frac{1}{2} a^{\prime 2}-\frac{1}{2} a^{\prime} b^{\prime}+a^{\prime} c^{\prime}+\frac{2}{r} a^{\prime}\right) \\
R_{r r}=-\frac{1}{2}\left(a^{\prime \prime}+2 c^{\prime \prime}\right)+\frac{b^{\prime}}{4}\left(a^{\prime}+2 c^{\prime}+\frac{4}{r}\right)-\frac{a^{\prime 2}}{4}-\frac{c^{\prime 2}}{2}-\frac{2}{r} c^{\prime} \\
R_{\vartheta \vartheta}=e^{c-b}\left[-1-\frac{r^{2}}{2} c^{\prime \prime}-r\left(2 c^{\prime}+\frac{a^{\prime}-b^{\prime}}{2}\right)-\frac{r^{2}}{4} c^{\prime}\left(a^{\prime}-b^{\prime}+2 c^{\prime}\right)\right]+1 \\
R_{\phi \phi}=\sin ^{2} \vartheta R_{\vartheta \vartheta}
\end{gathered}
$$

the prime always denotes $\partial / \partial r$. Then the Einstein's equations without matter can be reduced to the following three differential equations

$$
\begin{gathered}
G_{t t}=e^{a-b}\left[-c^{\prime \prime}-\frac{3}{4} c^{\prime 2}+\frac{1}{2} b^{\prime} c^{\prime}+\frac{1}{r}\left(b^{\prime}-3 c^{\prime}\right)\right]+\frac{1}{r^{2}}\left(e^{a-c}-e^{a-b}\right)=0 \\
G_{r r}=\frac{1}{2} a^{\prime} c^{\prime}+\frac{1}{r}\left(a^{\prime}+c^{\prime}\right)+\frac{c^{\prime 2}}{4}+\frac{1}{r^{2}}\left(1-e^{b-c}\right)=0 \\
G_{\vartheta \vartheta}=\frac{r^{2}}{2} e^{c-b}\left[a^{\prime \prime}+c^{\prime \prime}-\frac{1}{r}\left(b^{\prime}-a^{\prime}-2 c^{\prime}\right)+\frac{1}{2}\left(a^{\prime 2}-a^{\prime} b^{\prime}+a^{\prime} c^{\prime}-b^{\prime} c^{\prime}+c^{\prime 2}\right)\right]=0
\end{gathered}
$$

As usual $G_{\alpha \beta}$ is the Einstein tensor.

It is not hard to see that there are only two independent field equations. Indeed, using (3.6) $b$ can be expressed by $a$ and $c$. Eliminating $b$ in (3.5) and (3.7) there results one second order differential equation for $a$ and $c$ :

$$
c^{\prime \prime}=\frac{a^{\prime \prime}}{a^{\prime}}\left(c^{\prime}+\frac{2}{r}\right)+\frac{4}{r^{2}}+a^{\prime} c^{\prime}+\frac{c^{\prime 2}}{2}+\frac{2}{r}\left(a^{\prime}+c^{\prime}\right) .
$$

Introducing the new metric function

$$
f(r)=c(r)+2 \log r r_{c}
$$

where $r_{c}$ has been included for dimensional reasons, Equation (3.8) assumes the simple form

$$
\frac{f^{\prime \prime}}{f^{\prime}}-\frac{a^{\prime \prime}}{a^{\prime}}=a^{\prime}+\frac{f^{\prime}}{2}
$$

This can immediately by integrated

$$
\log \frac{f^{\prime}}{a^{\prime}}=a+\frac{f}{2}+\text { const. }
$$

On the other hand the circular velocity squared (2.14) becomes

$$
V^{2}(r) \equiv u=\frac{a^{\prime}}{f^{\prime}}
$$

It is the velocity squared $u(r)$ which appears in all equations. Using (3.12) in (3.11) we have

$$
a+\frac{c}{2}+\log \frac{r}{r_{c}}=-\log u .
$$

Differentiating and eliminating $c^{\prime}$ by means of (3.9) and (3.12) in the form 


$$
c^{\prime}=f^{\prime}-\frac{2}{r}=\frac{a^{\prime}}{u}-\frac{2}{r},
$$

we find

$$
a^{\prime}=-\frac{2 u^{\prime}}{1+2 u} .
$$

This gives the first diagonal element of the metric

$$
g_{t t}=e^{a}=\frac{K_{a}}{1+2 u}
$$

where $K_{a}$ is a constant of integration. Then from (3.13) we get

$$
-g_{\vartheta \vartheta} r^{-2}=e^{c}=\left(\frac{1+2 u}{u}\right)^{2} \frac{K_{c}}{r^{2}},
$$

where $K_{c}$ is another integration constant which contains $r_{c}$. Finally, exp $b$ follows from (3.6)

$$
-g_{r r}=e^{b}=K_{c}\left(\frac{u^{\prime}}{u^{2}}\right)^{2}(1+2 u) .
$$

We have succeeded in expressing the metric by the circular velocity squared $u(r)$. If we choose $u=r_{s} / 2\left(r-r_{s}\right)$, we recover the standard gauge $c=0$ and the Schwarzschild metric with the Schwarzschild radius

$$
r_{s}=2 \sqrt{K_{c}}
$$

That means the Schwarzschild metric is embedded in a class of nonstandard solutions. We emphasize that these solutions describe different physics because the corresponding circular velocities squared $u(r)$ are different.

Now comes a pitfall for the well informed reader. In the books (e.g. [3], Equation (8.1.4)) the line element (2.12) is transformed to the so-called "standard form" by redefining the radial coordinate as follows

$$
\bar{r}=r e^{c / 2}=\frac{1+2 u}{u} \sqrt{K_{c}}
$$

according to (3.17). With an additional scale transformation

$$
\bar{t}=t \sqrt{K_{a}}
$$

our metric (3.16-18) assumes the Schwarzschild form

$$
\mathrm{d} s^{2}=\left(1-\frac{r_{s}}{\bar{r}}\right) \mathrm{d} \bar{t}^{2}-\left(1-\frac{r_{s}}{\bar{r}}\right)^{-1} \mathrm{~d} \bar{r}^{2}-\bar{r}^{2}\left(\mathrm{~d} \vartheta^{2}+\sin ^{2} \vartheta \mathrm{d} \phi^{2}\right) .
$$

Mathematically the class of nonstandard solutions has collapsed to the Schwarzschild solution. What does this mean physically? As discussed in the introduction we reject to interpret the metric (3.22) physically. Instead we consider the observable $u(r)=V^{2}(r)$. Solving (3.20) for $u(r)$ we obtain

$$
u(r)=\frac{r_{s}}{2\left(\bar{r}-r_{s}\right)} \equiv \bar{u}(\bar{r})
$$

where (3.19) has been used. This is just the Schwarzschild expression in the new coordinate $\bar{r}$. Now it is clear what has been done: The new radius $\bar{r}$ has been chosen in such a way that the measured $u(r)$ becomes equal to the Schwarzschild expression $\bar{u}(\bar{r})$. Such a transformation is trivially possible, but it has no physical significance. We see that solutions that are equivalent under diffeomorphisms can be physically inequivalent. One may ask the question: What is the right physical radius, $r$ or $\bar{r}$ ? The astronomer must give the answer. If he would work with $\bar{r}$ then for every measured rotation curve, i.e. for every galaxy, he must define a new radial coordinate $\bar{r}$. This is not what he does. He always applies the same measuring procedure (for example measuring the apparent luminosity) to all galaxies, and this gives our radius $r$. After all in reality, the astronomer 
adds, the rotation curves in galaxies are not Schwarzschild [7].

As far as the vacuum equations are concerned we are not able to predict the circular velocity; it must be given. But then from (3.16-18) we are able to predict other observable quantities which can be computed from the metric, for example lensing data [8]. In this way the theory can be tested. Another test is investigated in the next section.

\section{Thin Material Disk with a Dark Halo}

We study a simple model of a spiral galaxy by assuming that the normal matter is concentrated in the equatorial plane $z=0$ with a singular density $\sim \delta^{1}(z)$. For this problem the theory of distribution valued curvature tensor is appropriate which is mainly due to Israel [9]. To be self-contained we give a simple derivation of the relations we need. Another reason to do this is the following: In nonstandard general relativity we do not use geometric relations involving the metric. Einstein's equation is the only basis, therefore, all derivations must be double checked. Let $S$ be a three-dimensional surface in 4-space where the metric tensor $g_{\mu v}$ is continuous but has finite jumps in the normal derivatives; the derivatives in the tangential directions are assumed to be continuous. In an admissible coordinate system let $S$ be described by the equation

$$
\varphi(x)=0
$$

and have the normal vector

$$
n_{\mu}=\frac{\partial \varphi}{\partial x^{\mu}}
$$

Then the finite discontinuities in the first partial derivatives of $g_{\mu v}$ are given by

$$
\left.\left[g_{\mu v, \sigma}\right] \equiv \frac{\partial g_{\mu v}}{\partial x^{\sigma}}\right|_{+}-\left.\frac{\partial g_{\mu v}}{\partial x^{\sigma}}\right|_{-}=n_{\sigma} b_{\mu v},
$$

where + and - mean the limiting values from both sides of $S$. This follows from the decomposition of the gradient into normal and tangential components. The corresponding jumps in the Christoffel symbols then are

$$
2\left[\Gamma_{\beta \gamma}^{\alpha}\right]=n_{\beta} b_{\gamma}^{\alpha}+n_{\gamma} b_{\beta}^{\alpha}-n^{\alpha} b_{\beta \gamma} .
$$

The Ricci tensor

$$
R_{\mu v}=\partial_{\alpha} \Gamma_{\mu \nu}^{\alpha}-\partial_{v} \Gamma_{\mu \alpha}^{\alpha}+\Gamma_{\alpha \beta}^{\alpha} \Gamma_{\mu \nu}^{\beta}-\Gamma_{\nu \beta}^{\alpha} \Gamma_{\alpha \mu}^{\beta}
$$

contains derivatives of $\Gamma$, consequently the finite jumps lead to singular contributions proportional to the delta distribution $\delta_{S}$ with support on $S$ according to

$$
\left.\partial_{\beta} \Gamma_{\mu v}^{\alpha}\right|_{\text {sing }}=\left[\Gamma_{\mu \nu}^{\alpha}\right] n_{\beta} \delta_{s} .
$$

Then it follows from (4.4) that

$$
\left.R_{\mu v}\right|_{\text {sing }}=\frac{1}{2}\left(-n^{\alpha} n_{\alpha} b_{\mu v}+n^{\alpha} \tilde{b}_{\mu \alpha} n_{v}+n^{\alpha} \tilde{b}_{\alpha v} n_{\mu}\right) \delta_{s}
$$

with

$$
\tilde{b}_{\beta}^{\alpha}=b_{\beta}^{\alpha}-\frac{1}{2} b \delta_{\beta}^{\alpha}, \quad b=g^{\mu v} b_{\mu v} .
$$

This is in agreement with Equation (2.14) of Taub [9], note that his convention for the Ricci tensor is the negative of our (4.5).

In the Einstein's equations these singular distribution must be compensated by a distribution valued energymomentum tensor

$$
\left.\left(R_{\mu v}-\frac{1}{2} R\right) g_{\mu v}\right|_{\text {sing }}=\kappa t_{\mu v} \delta_{S},
$$

where 


$$
R=g^{\alpha \beta} R_{\alpha \beta}, \quad \kappa=\frac{8 \pi G}{c^{2}} .
$$

If the jumps $b_{\mu v}$ of the normal derivatives of $g_{\mu v}$ are known, $t_{\mu v}$ can be calculated from (4.7) and (4.9):

$$
-2 \kappa t_{\mu v}=n^{2}\left(\left(g_{\mu}^{\sigma}-\frac{n^{\sigma} n_{\mu}}{n^{2}}\right)\left(g_{v}^{\tau}-\frac{n^{\tau} n_{v}}{n^{2}}\right)-\left(g_{\mu v}-\frac{n_{\mu} n_{v}}{n^{2}}\right)\left(g^{\sigma \tau}-\frac{n^{\sigma} n^{\tau}}{n^{2}}\right)\right) b_{\sigma \tau},
$$

where $n^{2}=n^{\alpha} n_{\alpha}$. This agrees with Equation (6-2) of Taub. The singular contribution (4.11) must be added to the regular energy-momentum tensor which renders the field equations fulfilled outside of the surface $S$.

Now we come to our simple galaxy model where the normal matter is concentrated in the plane $\vartheta=\pi / 2$ which is our singular surface $S$. Outside this plane we have vacuum with a dark halo as it is described by the nonstandard spherically symmetric solution (3.16-18). To have a simple representation of the plane $z=0$ and the corresponding delta-measure we go over to cylindrical coordinates $(t, R, z, \phi)$

$$
r^{2}=R^{2}+z^{2}, \quad z=r \cos \vartheta, \quad \sin \vartheta=\frac{R}{r} .
$$

Then the metric (2.12) assumes the following non-diagonal form

$$
\mathrm{d} s^{2}=g_{\mu v} \mathrm{~d} x^{\mu} \mathrm{d} x^{v}
$$

with

$$
\begin{aligned}
& g_{00}=e^{a}, \quad g_{11}=-\frac{R^{2}}{r^{2}} e^{b}-\frac{z^{2}}{r^{2}} e^{c}, \quad g_{22}=-\frac{R^{2}}{r^{2}} e^{c}-\frac{z^{2}}{r^{2}} e^{b}, \\
& g_{12}=g_{21}=-2 \frac{r z}{r^{2}}\left(e^{b}-e^{c}\right), \quad g_{33}=-R^{2} e^{c} .
\end{aligned}
$$

For simplicity we still write $r$, but our admissible coordinates are $x^{1}=R, x^{2}=z$. We also need the inverse

$$
g^{00}=e^{-a}, \quad g^{11}=\frac{g_{22}}{D}, \quad g^{22}=\frac{g_{11}}{D}, \quad g^{12}=-\frac{g_{12}}{D}=g^{21}, \quad g^{33}=\frac{1}{g_{33}},
$$

where the determinant $D$ is equal to

$$
D=g_{11} g_{22}-\left(g_{12}\right)^{2}=e^{b+c}-3 \frac{R^{2} z^{2}}{r^{4}}\left(e^{b}-e^{c}\right)^{2} .
$$

To construct the metric with the material disk we apply the widely used displace, cut, and reflect method which goes back to Kuzmin [10] and since then has been used by many authors. Following the procedure of Voigt and Letelier [11] we take the metric (3.7) in the half space $z>d>0$, displace it to $z=0$ and reflect it for $z<0$. This produces the finite jumps in the $z$-derivatives of $g_{\mu v}$. The whole procedure is equivalent to the transformation $z \rightarrow|z|+d$. The normal vector is $n_{\mu}=(0,0,1,0)=\delta_{\mu}^{2}$ and

$$
n^{v}=g^{v \mu} n_{\mu}=g^{v 2}, \quad n^{v} n_{v}=g^{22}
$$

The jumps (4.3) in the normal derivatives on $z=0$ which we need are equal to

$$
\begin{gathered}
b_{11}=\left[g_{11,2}\right]=g_{11}^{\prime} \frac{2 d}{r}-\frac{4 d}{r^{2} e^{c}} \\
b_{33}=\left[g_{33,2}\right]=g_{33}^{\prime} \frac{2 d}{r}
\end{gathered}
$$

where the prime always means $\partial / \partial r$ keeping $z$ and $R$ constant. Now from (4.11) we find the energy density

$$
t_{0}^{0}=\frac{1}{2} \kappa\left(D b_{11}+\frac{g_{11}}{D g_{33}} b_{33}\right)
$$

with $D=g_{11} g_{22}-g_{12}^{2}$. Using 


$$
b_{11}=\frac{2 d}{r}\left(g_{11}^{\prime}-\frac{2}{r} e^{c}\right), \quad b_{33}=\frac{2 d}{r} g_{33} c^{\prime},
$$

we finally obtain

$$
t_{0}^{0}=-\frac{d}{\kappa r}\left(e^{b+c} \frac{R^{6}}{r^{4}} \partial_{r}\left(\frac{e^{b}}{r^{2}}\right)+\frac{2 R^{4}}{r^{5}} e^{b+2 c}-\frac{r^{2}}{R^{2}} \partial_{r} e^{-c}\right) .
$$

Here we have to put $z=0$ everywhere which gives $r^{2}=R^{2}+d^{2}$.

Now we must specify the circular velocity squared $u(r)$ in order to fix the metric. We are particularly interested in the case of an asymptotically flat circular velocity which in the usual terminology corresponds to a dark halo. Therefore we assume $u(r)$ of the form

$$
u(r)=u_{\text {flat }}+\frac{u_{1}}{r}+O\left(r^{-2}\right)
$$

for large $r$. Then it follows from (3.16-18)

$$
e^{a}=K_{a}+O\left(r^{-1}\right), \quad e^{b}=\frac{L_{b}}{r^{4}}+O\left(r^{-5}\right), \quad e^{c}=\frac{L_{c}}{r^{2}}+O\left(r^{-3}\right)
$$

where by (3.17)

$$
L_{c} \sim u_{\text {flat }}^{-2}=V_{\text {flat }}^{-4} \text {. }
$$

Using this in (4.18) the leading order comes from the last term

$$
t_{0}^{0}=\frac{2 d}{\kappa L_{c}} \frac{r^{2}}{R^{2}}\left(1+O\left(R^{-1}\right)\right) .
$$

This is proportional to the density of normal matter because we consider a static energy-momentum tensor. Taking (4.21) into account we find that

$$
t_{0}^{0} \sim u_{\text {flat }}^{2} \sim V_{\text {flat }}^{4}(R)
$$

for large $R$. This is in accordance with the baryonic Tully-Fisher relation for galaxies [3] [4], which states that the total baryonic mass $M$ is proportional to $V_{\text {flat }}^{4}$. In fact, the contribution of the inner part $R<R_{1}$ of the disk can be made arbitrarily small compared to the outer part between $R_{1}<R<R_{2}$, say [12]. We emphasize that $M$ is obtained from $t_{0}^{0}$ by integrating with the Euclidean surface measure $R \mathrm{~d} R \mathrm{~d} \phi$, because this is what astronomers are doing when they determine $M$ from luminosity measurements. Our theory gives a very natural explanation of the Tully-Fisher relation which, otherwise, theoretically and observationally is somewhat mysterious.

The radial pressure $t_{r}^{r}$ vanishes because $G_{r r}$ (3.6) does not contain a second derivative. Therefore our model must be interpreted as a dust disk with purely azimuthal stresses. This is not very realistic and it remains to be investigated whether the Tully-Fisher relation is a generic property for more physical galaxy models.

\section{Concluding Remarks}

Our finding is that in the solar system the right gauge is $c(r)=0$, but on the galactic scale we have $c(r) \neq 0$. One would like to have a deeper understanding of this apparent paradox. One possible explanation is the following. At the very end general relativity must describe the solar system, the milky way, the local galaxy cluster etc. simultaneously. The division into separated subsystems is a misleading simplification. Keeping this in mind a continuous transition from $c$ approximately zero on small scales to $c \neq 0$ on the large is quite natural.

Obviously on small scales as the solar system or the binary pulsars the standard theory based on the geometric interpretation is the right one. But on the galactic scale which is a factor $10^{8}$ bigger, the non-geometric aspect of general relativity becomes visible. In both cases we are observing geodesics in a gravitational field. On the small scale this field can be described geometrically, on the large scale this is not the appropriate picture.

Our solutions in Section 3 seem to be the right ones to describe the dark halo of galaxies, if some dark matter cannot be found experimentally. The Tully-Fisher relation found in the last section is a central relation in modified Newtonian dynamics (MOND) [13]. This suggests that nonstandard GR is in accordance with MOND in 
contrast to standard GR. As far as the vacuum equations are concerned this is obviously true because nonstandard GR does not predict the circular velocity $V(r)$. The same remains true if we include normal matter in hydrostatic equilibrium [14]. We expect that the analysis of a detailed galaxy model in the framework of nonstandard GR will give the rotation curve $V(r)$. Indeed, the analysis of the last section shows that nonstandard GR solves the inverse problem: Given the rotation curve we can calculate the energy-momentum tensor. In standard GR the problem usually is posed the other way around. In the literature one has studied various modifications of GR to make MOND relativistic [15]. We have seen that this is not needed, nonstandard GR does the job.

\section{References}

[1] Poincaré, H. (1952) Science and Hypothesis. Dover Publications, Inc., New York.

[2] Xenon 100 Collaboration, 2012, arXiv 1207.5988

[3] Weinberg, S. (1972) Gravitation and Cosmology. John Wiley \& Sons, Hoboken.

[4] Tully, R.B. and Fisher, J.R. (1977) A New Method of Determining Distances to Galaxies. Astronomy and Astrophysics, 54, 661-673.

[5] McGaugh, S.S., Schombert, J.M., Bothun, G.D. and de Blok, W.J.G. (2000) The Baryonic Tully-Fisher Relation. The Astrophysical Journal, 533, L99-L102.

[6] Scharf, G. (2011) Dark Matter in Galaxies According to the Tensor-Four-Scalars Theory. Physical Review D, 84, Article ID: 084045. http://dx.doi.org/10.1103/PhysRevD.84.084045

[7] Corbelli, E. (2003) Dark Matter and Visible Baryons in $\mathrm{M}_{33}$. Monthly Notices of the Royal Astronomical Society, 342, 199-207. http://dx.doi.org/10.1046/j.1365-8711.2003.06531.x

[8] Bräunlich, G. and Scharf, G. (2010) Gravitational Lensing and Rotation Curve. General Relativity and Gravitation, 43, 143-154.

[9] Taub, A.H. (1980) Space-Times with Distribution-Valued Curvature Tensors. Journal of Mathematical Physics, 21, 1423.

[10] Kuzmin, G.G. (1956) Astron. Zh., 33, 27

[11] Voigt, D. and Letelier, P.S. (2003) Exact General Relativistic Perfect Fluid Disks with Halos. Physical Review D, 68, Article ID: 084010. http://dx.doi.org/10.1103/PhysRevD.68.084010

[12] Scharf, G. (2012) Dark Matter in Galaxies According to the Tensor-Four-Scalars Theory III. arXiv 1205.4309.

[13] Milgrom, M. (1983) A Modification of the Newtonian Dynamics-Implications for Galaxy Systems. Astrophysical Journal, 270, 384. http://dx.doi.org/10.1086/161132

[14] Scharf, G. (2012) Nonstandard General Relativity II. arXiv 1210.1496.

[15] Bekenstein, J.D. (2004) Revised Gravitation Theory for the Modified Newtonian Dynamics Paradigm. Physical Review $D$, 70, Article ID: 083509. 